\title{
Recurrent BRAF Gene Fusions in a Subset of Pediatric Spindle cell Sarcomas - Expanding the Genetic Spectrum of Tumors with Overlapping Features with Infantile Fibrosarcoma
}

\author{
Yu-Chien Kao, MD ${ }^{1,2}$, Christopher D.M. Fletcher, MD, FRCPath ${ }^{3}$, Rita Alaggio, MD ${ }^{4}$, Leonard \\ Wexler, MD ${ }^{5}$, Lei Zhang, MD ${ }^{1}$, Yun-Shao Sung, MSc $^{1}$, Dicle Orhan, MD $^{6}$, Wei-Chin Chang, \\ MD $^{7,8,9}$, David Swanson ${ }^{10}$, Brendan C Dickson, MD ${ }^{10}$, and Cristina R Antonescu, MD ${ }^{1,{ }^{*}}$ \\ ${ }^{1}$ Department of Pathology, Memorial Sloan Kettering Cancer Center, New York, NY, USA \\ ${ }^{2}$ Department of Pathology, Shuang Ho Hospital, Taipei Medical University, Taipei, Taiwan \\ ${ }^{3}$ Department of Pathology, Brigham and Women's Hospital, Harvard Medical School, Boston, MA, \\ USA \\ ${ }^{4}$ Department of Pathology, Children's Hospital UPMC Pittsburgh, PA, USA \\ ${ }^{5}$ Department of Pediatrics, Memorial Sloan Kettering Cancer Center, New York, NY, USA \\ ${ }^{6}$ Department of Pathology, Hacettepe University, Ankara, Turkey \\ ${ }^{7}$ Department of Pathology, MacKay Memorial Hospital, Taipei, Taiwan \\ ${ }^{8}$ Department of Pathology, MacKay Medical College, New Taipei City, Taiwan \\ ${ }^{9}$ Department of Pathology, MacKay Medicine, Nursing, and Management College, New Taipei \\ City, Taiwan \\ ${ }^{10}$ Department of Pathology \& Laboratory Medicine, Mount Sinai Hospital, Toronto, Canada
}

\begin{abstract}
Infantile fibrosarcomas (IFS) represent a distinct group of soft tissue tumors occurring in patients under two years of age and most commonly involving the extremities. Most IFS show recurrent ETV6-NTRK3 gene fusions, sensitivity to chemotherapy, and an overall favorable clinical outcome. However, outside these well-defined pathologic features, no studies have investigated IFS lacking ETV6-NTRK3 fusions, or tumors with the morphology resembling IFS in older children. This study was triggered by the identification of a novel SEPT7-BRAFfusion in an unclassified retroperitoneal spindle cell sarcoma in a 16 year-old female by targeted RNA sequencing. FISH screening of nine additional tumors with similar phenotype and lacking ETV6NTRK 3 identified four additional cases with $B R A F$ gene rearrangements in the pelvic cavity $(\mathrm{n}=2)$, paraspinal region $(\mathrm{n}=1)$, and thigh $(\mathrm{n}=1)$ of young children ( $0-3$ years old). Histologically, four cases including the index case shared a fascicular growth of packed monomorphic spindle cells, with uniform nuclei and fine chromatin, and a dilated branching vasculature; while the
\end{abstract}

*Corresponding Author: Cristina R Antonescu, MD, Memorial Sloan-Kettering Cancer Center, Department of Pathology, 1275 York Ave, New York, NY, 10065; Phone: (212) 639-5905; antonesc@mskcc.org.

Conflicts of interest: none 
remaining case was composed of compact cellular sheets of short spindle to ovoid cells. In addition, a minor small blue round cell component was present in one case. Mitotic activity ranged from 1-9/10 high power fields. Immunohistochemical stains were non-specific, with only focal smooth muscle actin staining demonstrated in three cases tested. Of the remaining five $B R A F$ negative cases, further RNA sequencing identified one case with EML4-NTRK3 in an 1 year-old boy with a foot IFS, and a second case with TPM3-NTRK1 fusion in a 7 week-old infant with a retroperitoneal lesion. Our findings of recurrent $B R A F$ gene rearrangements in tumors showing morphologic overlap with IFS expand the genetic spectrum of fusion-positive spindle cell sarcomas, to include unusual presentations, such as older children and adolescents and predilection for axial location, thereby opening new opportunities for kinase-targeted therapeutic intervention.

\section{Keywords}

fibrosarcoma; infantile fibrosarcoma; $B R A F, N T R K 1 ; N T R K 3$; fusions

\section{INTRODUCTION}

Infantile fibrosarcomas (IFS) are malignant soft tissue neoplasms typically occurring congenitally, or within the first two years of life. Tumors most commonly affect the extremities and less often involve the trunk and head and neck regions. ${ }^{1,2}$ Histologically, IFS are hypercellular tumors composed of fascicles of monomorphic and often primitiveappearing spindle cells with scant cytoplasm. Additional findings include a hemangiopericytoma-like vascular pattern, a variable chronic inflammatory infiltrate and extramedullary hematopoiesis. ${ }^{1}$ Less common patterns include poorly-formed fascicles and areas of round cell histology. ${ }^{1}$ IFS is characterized by an indolent clinical course, with low risk of distant metastasis and a 5-year survival rate of $89 \% \cdot{ }^{1-3}$ Additionally these tumors show significant sensitivity to cytotoxic chemotherapy and more recently a promising response to NTRK inhibitors. ${ }^{2,4}$ Thus neoadjuvant regimens might be used in inoperable cases or to avoid mutilating surgery.

ETV6-NTRK3 gene fusions are the genetic hallmark of IFS and are present in the majority of cases. ${ }^{5}$ As IFS lack a specific immunoprofile, molecular confirmation of the prototypic $\mathrm{t}(12 ; 15)$ translocation, either by fluorescence in situ hybridization (FISH) or reverse transcription-polymerase chain reaction (RT-PCR), has become the gold-standard in routine diagnosis. ${ }^{6-8}$ However, a small subset of IFS lack the ETV6-NTRK3 fusion and have not yet been investigated for alternative genetic abnormalities. ${ }^{2,9}$ Furthermore, it remains unclear whether tumors resembling fibrosarcoma in older children are pathogenetically related to IFS or adult fibrosarcomas, or perhaps an independent pathologic entity, with as yet undefined genetic signatures. In this study, we report recurrent $B R A F$ gene fusions in five cases of spindle cell sarcoma morphologically resembling IFS in pediatric patients ranging from 2 days to 16 years of age and with an apparent predilection for axial locations. 


\section{MATERIALS AND METHODS}

\section{Index case and patient selection}

The index case was a 16 year-old female presenting with a retroperitoneal tumor, complicated by rupture and intra-abdominal hemorrhage. Intra-operatively the tumor showed peritoneal involvement and ureteral encasement. Morphologically, the tumor was composed of long intersecting fascicles of relatively uniform spindle cells with fine chromatin, mild nuclear atypia and scant fibrillary cytoplasm, associated with scattered lymphoplasmacytic infiltrate and dilated vascular channels (Fig. 1A-C). Focal infarction was present. A mitotic count was up to $6 / 10$ high power fields. The immunophenotype was non-specific, with only patchy staining for smooth muscle actin (Fig. 1D) and caldesmon. Other markers, including desmin, S100, c-kit, and DOG1 were negative. H3K27me3 expression was retained. FISH tests were negative for $S S 18, A L K, R O S 1$ and RET gene abnormalities. Based on these results a diagnosis of unclassified low grade spindle cell sarcoma was rendered and formalin-fixed paraffin-embedded (FFPE) tissue was submitted for targeted RNA sequencing for potential fusion discovery. After the identification and validation of the fusion candidate in the index case, we screened nine additional spindle cell sarcomas in children (cases \#2-10, Table 1), selected based on a similar primitive phenotype, lack of a specific line of differentiation by immunohistochemistry and absence of canonical ETV6-NTRK3 fusion. The study was approved by the Institutional IRB.

\section{Targeted RNA sequencing}

The index case and case \#6 were submitted for targeted RNA sequencing. RNA was extracted from FFPE tissue using Amsbio's ExpressArt FFPE Clear RNA Ready kit (Amsbio LLC, Cambridge, MA). Fragment length was assessed with an RNA 6000 chip on an Agilent Bioanalyzer (Agilent Technologies, Santa Clara, CA). RNA-seq libraries were prepared using 20-100 ng total RNA with the Trusight RNA Fusion Panel (Illumina, San Diego, CA). Targeted RNA sequencing was performed on an Illumina MiSeq platform. Reads were independently aligned with STAR(ver 2.3) against the human reference genome (hg19) and analyzed by STAR-Fusion.

\section{Whole transcriptome sequencing}

Case \#7 in the screening cohort had available frozen tissue and was submitted for whole transcriptome sequencing. Total RNA was extracted from frozen tissues using RNeasy Plus Mini (Qiagen), followed by mRNA isolation with oligo(dT) magnetic beads and fragmentation by incubation at $94^{\circ} \mathrm{C}$ in fragmentation buffer (Illumina) for 2.5 minutes. After gel size-selection (350-400bp) and adapter ligation, the library was enriched by PCR for 15 cycles and purified. Paired-end RNA sequencing at read lengths of 50 or 51 bp was performed with the HiSeq 2000 (Illumina).

\section{Fluorescence in situ hybridization (FISH)}

To validate the fusion candidates found by RNA sequencing, we performed FISH for $B R A F$ break-apart and SEPT7-BRAFfusion assay in the index case; EML4 and NTRK3 breakapart in case \#6; and TPM3-NTRK1 fusion assay in case \#7. Other cases were screened by 
FISH for $B R A F, N T R K 3$, and NTRK1 gene abnormalities. Custom probes were made by bacterial artificial chromosomes (BAC) clones flanking $B R A F$ according to UCSC genome browser (http://genome.ucsc.edu) and obtained from BACPAC sources of Children's Hospital of Oakland Research Institute (Oakland, CA; http://bacpac.chori.org) (Supplementary Table 1). The BAC representing SEPT7 (RP11-1011L9) covered part of the gene and its centromeric region. DNA from each BAC was isolated according to the manufacturer's instructions. The BAC clones were labeled with fluorochromes (fluorescentlabeled dUTPs, Enzo Life Sciences, NY, USA) by nick translation and validated on normal metaphase chromosomes. The $4 \mu \mathrm{m}$-thick FFPE slides were deparaffinized, pretreated, and hybridized with denatured probes. After overnight incubation, the slides were washed, stained with 4',6-diamidino-2-phenylindole (DAPI), mounted with an antifade solution, and then examined on a Zeiss fluorescence microscope (Zeiss Axioplan, Oberkochen, Germany) controlled by Isis 5 software (Metasystems).

\section{Reverse transcription-polymerase chain reaction (RT-PCR)}

RT-PCR was performed to validate the fusion transcript identified by RNA sequencing in the index case and case \#7. For the index case, RNA was extracted from FFPE tissues using RNeasy FFPE (Qiagen) and reverse transcribed by SuperScript IV First-Strand Synthesis System (Invitrogen). For case \#7, the remaining RNA from the RNA sequencing was reverse-transcribed by SuperScript III First-Strand Synthesis System (Invitrogen). PCR was performed by Advantage 2 PCR kit (Clontech, Mountain View, CA). For the index case, the reaction was run at $67^{\circ} \mathrm{C}$ annealing temperature for 38 cycles with the following primers: forward - SEPT7 exon 11 (5'-GAGCAGGTGTTTGAGATGAAGGTC) and reverse - BRAF exon 11 (5'-CCACTTTCCCTTGTAGACTGTTCC). For case \#7, the reaction was run at $64.5^{\circ} \mathrm{C}$ annealing temperature for 33 cycles with the following primers: forward - TPM3 exon 5 (5'- CTGAAGTGTCTGAGTGCTGCTGAAG) and reverse - NTRK1 exon 10 (5'GGAAGAGGCAGGCAAAGACG). The PCR product was analyzed by gel electrophoresis and Sanger sequencing.

\section{RESULTS}

\section{A novel SEPT7-BRAF fusion identified in an unclassified spindle cell sarcoma reminiscent of IFS}

Targeted RNA sequencing identified a SEPT7-BRAFfusion candidate in the index case. The fusion resulted from a pericentric inversion of chromosome 7, leading to an in-frame fusion of exon 11 of $S E P T 7$ (7p14.2) to exon 11 of $B R A F$ (7q34), as confirmed by RT-PCR (Fig. 2 A, B). The kinase domain of BRAF, encoded by exons 11 to 18 was retained in the predicted fusion protein. The reciprocal $B R A F-S E P T 7$ fusion junction reads were also identified in the RNA sequencing data. The $S E P T 7-B R A F$ fusion was further confirmed by FISH showing a $B R A F$ break-apart signal as well as by FISH fusion assay showing the $B R A F$ and $S E P T 7$ signals come-together (Fig. 2 C). BRAFmRNA expression level was not significantly upregulated compared to other soft tissue tumor samples available on the same RNA sequencing platform (data not shown); this is in keeping with the expression levels of $B R A F$ fusions in other soft tissue tumors, melanomas or carcinomas. ${ }^{10}$ 


\section{Recurrent BRAF gene rearrangements identified in four additional IFS}

We identified 9 additional pediatric sarcoma cases showing features indistinguishable from IFS morphologically, with an age range of $0-12$ years old, located in the abdominal cavity ( 2 each for pelvis and retroperitoneum), trunk (1 each for paraspinal and lumbar), and extremity ( 1 each for thigh, foot, and forearm). All cases showed a non-specific immunoprofile and were negative for the ETV6-NTRK 3 fusion. FISH screening for $B R A F$ gene abnormalities identified 4 additional cases positive for rearrangements (cases \#2-5, Supplementary Fig. 1 A, B). Similar to the index case, cases \#2 and \#3 occurred within the abdominal cavity. Case \#2 was a 3 year-old girl presenting with a pelvic mass, in close vicinity to the uterus, bladder and ureters and was attached to the presacral fascia on the posterior aspect. Case \#3 was a 6 month-old girl with a pelvic soft tissue mass. The other 2 $B R A F$-rearranged tumors were in somatic soft tissues. Case \#4 was an extradural tumor involving T6 to T12 including bilateral neural foramina. The patient presented with extremity weakness, truncal instability, and refusal to walk. Unlike ETV6-NTRK3 rearranged infantile fibrosarcomas, which frequently involve extremities, only 1 of the $B R A F$ rearranged cases occurred in the extremity, presenting as a congenital mass in the thigh, extending into pelvis (case \#5). Morphologically, 3 tumors showed monomorphic spindle cells arranged in intersecting fascicles accompanied by scattered dilated vascular spaces (Fig. 3 A-E, H), while the remaining case \#4 showed compact sheets of short spindle to ovoid tumor cells (Fig. 3G). A focal round cell component was also identified in case \#3 (Fig. 3 F). The tumor cells had a primitive appearance, with fine chromatin and scant cytoplasm. Infiltrative growth into immature skeletal muscle was noted in the congenital case (case \#5, Fig. 3I). Mitotic activities ranged from 1 to 9 mitoses per 10 high power fields. Focal necrosis was seen in case \#5. Immunohistochemically, cases \#3 and \#5 showed focal positivity for SMA, while other immunostains were non-contributory. Case \#4 showed positivity for ETV4 and WT1 staining, but no $C I C$ gene abnormalities were noted by FISH. Independent to our investigation, case \#4 was found to have a $C U X 1-B R A F$ fusion by FoundationOne sequencing report, which confirmed our FISH result. The fusion partners remained unknown for the remaining 3 cases lacking RNA material for further study. Fusion FISH assay for SEPT7-BRAFfusion was negative in case \#2, while non-contributory in case \#3 due to a deletion of the telomeric signal of $B R A F$.

\section{Alternative EML4-NTRK3 and TPM3-NTRK1 gene fusions identified in 2 additional IFS cases}

Case \#6 occurred in a 21 month-old male who presented with a $4 \mathrm{~cm}$ foot mass, involving the extensor tendons of $4^{\text {th }}-5^{\text {th }}$ digits. The lump had been present for over a year, and had increased in size in the prior 2-3 months. Histologically, the tumor was composed of round, ovoid and short spindle cells arranged in vague nests and short fascicles, associated with areas of necrosis. The lesional cells showed scant eosinophilic cytoplasm and monomorphic nuclei with fine chromatin (Fig. 6A,B). The mitotic activity was brisk of $>10 \mathrm{MF} / 10 \mathrm{HPFs}$. Immunohistochemically, the tumor showed CD34 positivity, while being negative for S100, CK, desmin and myogenin. Targeted RNA sequencing identified an EML4-NTRK3 fusion, in which exon 2 of EML4 was fused to exon 14 of NTRK3 (Fig. 4A,B). This breakpoint was identical to the only other reported IFS tumor with EML4-NTRK3 fusion occurring in an 
upper extremity of a 9 month-old boy. ${ }^{11}$ This result was further confirmed by FISH (Fig. $4 C)$.

Case \#7 was that of a 7 week-old boy presenting with a $12 \mathrm{~cm}$ retroperitoneal tumor, which was removed piece-meal. He subsequently developed 2 local recurrences that were removed surgically, 1 year and 4 years after the first excision, respectively. At the time of $2^{\text {nd }}$ recurrence he was also diagnosed with lung metastases. The patient was alive with stable primary and metastatic lesions 11 years after the initial diagnosis. The tumor was negative for ETV6-NTRK3 fusion by RT-PCR and was diagnosed as a fusion-negative IFS. Frozen tumor was available and subjected to whole transcriptome sequencing which showed a TPM3-NTRK1 fusion candidate (Fig 5A,B). RT-PCR confirmed the fusion of exon 6 of TPM3 with exon 9 of NTRK1, which preserves the C-terminal kinase domain of NTRK1, similar to the other 2 IFS cases with NTRK1 fusions reported in the literature, although the fusion partners and exact breakpoint of NTRK1 is variable, with SQSTM1 exon 2 - NTRK1 exon 10 and $L M N A$ exon 10 - NTRK1 exon 12, resepctively. ${ }^{12,13}$ Further FISH testing confirmed rearrangement of NTRK1 as well as fusion with TPM3 (Fig. 5C). NTRK1 mRNA expression showed up-regulation of downstream exons included in the fusion transcript (Fig. 5D), which correlated with NTRK1 cytoplasmic expression by immunohistochemistry. Morphologically, it showed a monomorphic spindle cell neoplasm arranged in long, intersecting fascicles and prominent cleft-like vasculature (Fig. 6C,D). The tumor cells showed fine chromatin with focal mild cytologic atypia and a mitotic activity of $5 \mathrm{MF} / 10$ HPFs. Immunohistochemically, the tumor cells were positive for SMA and CD34. The remaining 3 cases were negative for $B R A F, N T R K 3$, and $N T R K 1$ rearrangements by FISH.

\section{DISCUSSION}

We investigated a group of 10 ETV6-NTRK3 fusion negative unclassified spindle cell sarcomas with overlapping features of infantile fibrosarcoma (IFS) occurring in the pediatric age group, for further molecular classification. Our combined approach, using RNA sequencing for fusion gene discovery and FISH screening, revealed recurrent $B R A F$ gene rearrangements in 5 cases of spindle cell sarcomas, morphologically akin to IFS, occurring within the abdominal cavity, paraspinal region, and thigh of children, ranging from 0 to 16 year-old. The morphologic appearance was characterized by primitive/ monomorphic spindle cells arranged in long, intersecting fascicles, associated with a variable hemangiopericytoma-like vascular pattern. More ovoid to round cytomorphology was also seen. The tumors showed no specific line of differentiation by immunohistochemistry, with only focal SMA reactivity seen in 3 cases tested. The wide age range at diagnosis, spanning infancy to adolescence age, raises the question whether this molecular group of tumors represents a new pathologic entity or shares a pathogenetic link with NTRK3-fusion positive IFS. Furthermore, $3 B R A F$ rearranged tumors occurred within the abdominal cavity (one retroperitoneum, two pelvic). Intra-abdominal or retroperitoneal locations are uncommon in IFS, comprising about $12 \%$ of patients in a recent large series. ${ }^{14}$ Although some intraabdominal IFS do harbor ETV6-NTRK3 fusions, a case series of 4 IFS involving ileum or jejunum in newborns were negative for the fusion in all the 3 cases tested, raising the possibility that a subset of intestinal or intra-abdominal IFS may either be enriched for alternative gene fusions or represent a distinct pathologic entity. ${ }^{15}$ 
The genetic hallmark of IFS is the presence of recurrent ETV6-NTRK3 gene fusion, which is also demonstrated in the cellular congenital mesoblastic nephroma (CMN) of the kidney, suggesting a common pathogenesis. ${ }^{16}$ However, a subset of pediatric spindle cell sarcomas morphologically similar to IFS lack the ETV6-NTRK3 gene fusion and are either regarded as fusion-negative IFS or grouped by default into an unclassified spindle cell sarcoma category. The latter terminology implies a high grade behavior, especially when being mitotically active as in some of our cases, and thus might trigger a reflex neoadjuvant therapy with the use of chemoradiation, even in surgically amenable cases. And, although the experience remains limited due to small number of cases, the emerging data suggests that other fusion-positive congenital or infantile spindle cell sarcomas, besides IFS, such as VGLL2-fusion positive spindle cell rhabdomyosarcoma, ${ }^{17}$ might follow a similarly favorable outcome, in which a surgically removed lesion might not require intensive adjuvant therapy.

In a series of 14 IFS/CMN, Gadd et al. identified 4 cases negative for ETV6-NTRK3 fusions. ${ }^{9}$ Regardless of their fusion status the IFS/CMN showed distinctive mRNA and protein expression profiles and by unsupervised hierarchical clustering the fusion-negative IFS/CMN clustered together with ETV6-NTRK3-positive IFS/CMN, compared to other soft tissue tumors in the same dataset. Among the differentially expressed genes, the IFS/CMN showed upregulation of $E G R 1$ and downregulation of $F G F R 2$, an expression pattern which was also found in our index case with $S E P T 7-B R A F$ fusion.

$B R A F$ related fusions have been reported in various tumors including gliomas, carcinomas, and melanocytic neoplasms through either intra- or inter-chromosomal translocations. ${ }^{18-22}$ Among soft tissue tumors, $B R A F$ gene rearrangements were only recently reported in a subset of myxoinflammatory fibroblastic sarcomas, a clinically and morphologically distinct tumor type, characterized by superficial acral location and composed of alternating myxoid and solid areas, with a histiocytoid phenotype and Reed-Sternberg-like tumor cells. ${ }^{10} \mathrm{In}$ addition, a case of a chest wall malignant spindle cell neoplasm harboring a KIAA1549$B R A F$ fusion was reported in an adult patient with distinct morphology. ${ }^{23}$ From the publicly available expression data, neither $B R A F$-fusion positive carcinomas nor sarcomas appear to be associated with a $B R A F \mathrm{mRNA}$ upregulation. Therefore, immunohistochemical staining for wild-type BRAF is less likely to serve as a screening tool.

With the recent advent of next generation sequencing, a handful of IFS and related pediatric spindle cell sarcoma cases have been reported to harbor alternative gene fusions, either NTRK 3 fused to different 5' partner genes, or other kinases, such as NTRK1 and $M E T{ }^{11-13,24}$ An EML4-NTRK3 fusion resulting from a t(2;15) translocation was detected by cytogenetics, array comparative genomic hybridization, and RNA sequencing in a 9 month-old boy with an IFS of the left upper extremity. ${ }^{11}$ The patient developed lung metastasis which responded to chemotherapy and radiation therapy. The tumorigenic potential of EML4-NTRK3 was also demonstrated by anchorage-independent growth of NIH $3 \mathrm{~T} 3$ cells expressing the fusion transcript and in vivo tumor growth. Similar to their patient, our case was also located in the extremity (foot) and had an identical exonic composition of the fusion transcript. Histologically, their case was composed of elongated spindle cells and was CD34 negative, while our case had a more primitive appearance 
comprised of round to short spindle cells and immunopositivity for CD34. Both cases showed a brisk mitotic activity (>10/10HPFs). Two additional IFS cases identified by targeted DNA and RNA sequencing at Foundation Medicine harbored SQSTM1-NTRK1 and $L M N A-N T R K 1$ fusion transcripts, respectively, although no histologic description or images were provided. ${ }^{12,13}$ The latter case presented as a buttock mass in a male infant, who subsequently developed local recurrence and metastasis to bilateral lungs and S5 vertebral body. The metastatic lesions were refractory to chemotherapy but responded to crizotinib. ${ }^{13}$ Although the exonic composition of the 3 IFS cases with NTRK1 fusions is slightly variable, including exon 9 (our case, TPM3-NTRK1), exon 10 (SQSTM1-NTRK1), or exon 12 ( $L M N A-N T R K 1)$, they all retain the full-length coding sequence of the tyrosine kinase domain of NTRK1 (exons 13-17). Furthermore, NTRK1-related gene fusions, including an identical TPM3-NTRK1 fusion, were identified in two distinct soft tissue tumor types, including lipofibromatosis-like neural tumors and a group of spindle cell sarcomas with prominent myopericytic/haemangiopericytic pattern. ${ }^{25,26}$ Both these entities have distinct morphologic features from IFS, lacking the primitive monomorphic spindle cells arranged in long fascicles. Furthermore, the so-called lipofibromatosis-like neural tumor is characterized by a highly infiltrative growth pattern within adipose tissue and shows co-expression of CD34 and S100 protein immunohistochemically. ${ }^{25}$ More recently, our group identified a novel TFG-MET gene fusion by RNA sequencing in a retroperitoneal spindle cell sarcoma in a 4 month-old female showing strong S100 protein expression suggestive of neural features. ${ }^{24}$ The patient was treated by incomplete surgical resection followed by chemotherapy and remains clinically stable.

$S E P T 7$, identified in our index case is a novel fusion partner to $B R A F$, belongs to the septin gene family; it has GTPase activity and is associated with cytoskeleton structure and organelle transport. ${ }^{27}$ Another member of the septin gene family, SEPT9, was previously reported in a case of T-cell prolymphocytic leukemia with SEPT9-ABL1 fusion, which was resistant to both imatinib and dasatinib therapy. ${ }^{28}$ Additionally, other members of the septin family, including SEPT2, SEPT5, SEPT6, SEPT9, and SEPT11, were involved as 3' partners in hematological malignancies with $M L L-S E P T I N$ genes fusions. ${ }^{29}$

In conclusion, our findings of recurrent $B R A F$-gene fusions in a group of tumors with features reminiscent of IFS expand the spectrum of fusion-positive spindle cell sarcomas to also include older children and adolescents and predilection for intra-abdominal sites. We also describe a $2^{\text {nd }}$ case of IFS with EML4-NTRK3 fusion and a $3^{\text {rd }}$ example of IFS with NTRK1 fusion, with a previously unreported gene partner in this tumor type, TPM3. These findings bring further foundation to an increasing number of primitive sarcomas of childhood driven by oncogenically activated kinases through gene fusions, with critical therapeutic implications. Previous reports demonstrated promising sensitivity to LOXO-101, an inhibitor of TRK family, in ETV6-NTRK3 fused IFS, ${ }^{4}$ as well as clinical responses of NTRK1-rearranged IFS to crizotinib. ${ }^{13}$ Therefore, in the setting of pediatric tumors with infantile fibrosarcoma-like phenotype, the molecular work-up should ideally include testing for abnormalities in other kinases, such as $B R A F, N T R K 1, M E T$, etc, if the tumor lacks the canonical ETV6-NTRK3 fusion. Further studies are needed to establish if the $B R A F-$ rearranged tumors showing phenotypic overlap with IFS but occurring in unusual clinical settings, such as older age group or intra-abdominal location, are associated with a similarly 
favorable outcome, or represent a stand-alone pathologic entity with a different biologic behavior. Larger series of pediatric spindle cell sarcomas characterized by gene fusions across various kinases will also provide insight on their shared morphologic and clinical features, as well as their responses to targeted therapy.

\section{Supplementary Material}

Refer to Web version on PubMed Central for supplementary material.

\section{Acknowledgments}

Supported in part by: P50 CA140146-01 (CRA); P30-CA008748 (CRA); Kristen Ann Carr Foundation (CRA); Cycle for Survival (CRA)

\section{References}

1. Chung EB, Enzinger FM. Infantile fibrosarcoma. Cancer. 1976; 38:729-739. [PubMed: 974993]

2. Orbach D, Rey A, Cecchetto G, et al. Infantile fibrosarcoma: management based on the European experience. J Clin Oncol. 2010; 28:318-323. [PubMed: 19917847]

3. Soule EH, Pritchard DJ. Fibrosarcoma in infants and children: a review of 110 cases. Cancer. 1977; 40:1711-1721. [PubMed: 561651]

4. Nagasubramanian R, Wei J, Gordon P, et al. Infantile Fibrosarcoma With NTRK3-ETV6 Fusion Successfully Treated With the Tropomyosin-Related Kinase Inhibitor LOXO-101. Pediatr Blood Cancer. 2016; 63:1468-1470. [PubMed: 27093299]

5. Knezevich SR, McFadden DE, Tao W, et al. A novel ETV6-NTRK3 gene fusion in congenital fibrosarcoma. Nat Genet. 1998; 18:184-187. [PubMed: 9462753]

6. Adem C, Gisselsson D, Dal Cin P, et al. ETV6 rearrangements in patients with infantile fibrosarcomas and congenital mesoblastic nephromas by fluorescence in situ hybridization. Mod Pathol. 2001; 14:1246-1251. [PubMed: 11743047]

7. Argani P, Fritsch M, Kadkol SS, et al. Detection of the ETV6-NTRK3 chimeric RNA of infantile fibrosarcoma/cellular congenital mesoblastic nephroma in paraffin-embedded tissue: application to challenging pediatric renal stromal tumors. Mod Pathol. 2000; 13:29-36. [PubMed: 10658907]

8. Bourgeois JM, Knezevich SR, Mathers JA, et al. Molecular detection of the ETV6-NTRK3 gene fusion differentiates congenital fibrosarcoma from other childhood spindle cell tumors. Am J Surg Pathol. 2000; 24:937-946. [PubMed: 10895816]

9. Gadd S, Beezhold P, Jennings L, et al. Mediators of receptor tyrosine kinase activation in infantile fibrosarcoma: a Children's Oncology Group study. J Pathol. 2012; 228:119-130. [PubMed: 22374738]

10. Kao YC, Ranucci V, Zhang L, et al. Recurrent BRAF Gene rearrangements in Myxoinflammatory fibroblastic sarcomas, but not hemosiderotic fibrolipomatous tumors. Am J Surg Pathol. 2017 [In Press].

11. Tannenbaum-Dvir S, Glade Bender JL, Church AJ, et al. Characterization of a novel fusion gene EML4-NTRK3 in a case of recurrent congenital fibrosarcoma. Cold Spring Harb Mol Case Stud. 2015; 1:a000471. [PubMed: 27148571]

12. Pavlick D, Schrock AB, Malicki D, et al. Identification of NTRK fusions in pediatric mesenchymal tumors. Pediatr Blood Cancer. 2017

13. Wong V, Pavlick D, Brennan T, et al. Evaluation of a Congenital Infantile Fibrosarcoma by Comprehensive Genomic Profiling Reveals an LMNA-NTRK1 Gene Fusion Responsive to Crizotinib. J Natl Cancer Inst. 2016:108.

14. Sulkowski JP, Raval MV, Browne M. Margin status and multimodal therapy in infantile fibrosarcoma. Pediatr Surg Int. 2013; 29:771-776. [PubMed: 23765394]

15. Berrebi D, Fournet JC, Boman F, et al. Intestinal congenital/infantile fibrosarcoma: a new clinicopathological entity? Pediatr Surg Int. 2015; 31:375-379. [PubMed: 25652760] 
16. Rubin BP, Chen CJ, Morgan TW, et al. Congenital mesoblastic nephroma t $(12 ; 15)$ is associated with ETV6-NTRK3 gene fusion: cytogenetic and molecular relationship to congenital (infantile) fibrosarcoma. Am J Pathol. 1998; 153:1451-1458. [PubMed: 9811336]

17. Alaggio R, Zhang L, Sung YS, et al. A Molecular Study of Pediatric Spindle and Sclerosing Rhabdomyosarcoma: Identification of Novel and Recurrent VGLL2-related Fusions in Infantile Cases. Am J Surg Pathol. 2016; 40:224-235. [PubMed: 26501226]

18. Jones DT, Kocialkowski S, Liu L, et al. Tandem duplication producing a novel oncogenic BRAF fusion gene defines the majority of pilocytic astrocytomas. Cancer Res. 2008; 68:8673-8677. [PubMed: 18974108]

19. Wiesner T, He J, Yelensky R, et al. Kinase fusions are frequent in Spitz tumours and spitzoid melanomas. Nat Commun. 2014; 5:3116. [PubMed: 24445538]

20. Ciampi R, Knauf JA, Kerler R, et al. Oncogenic AKAP9-BRAF fusion is a novel mechanism of MAPK pathway activation in thyroid cancer. J Clin Invest. 2005; 115:94-101. [PubMed: 15630448]

21. Chmielecki J, Hutchinson KE, Frampton GM, et al. Comprehensive genomic profiling of pancreatic acinar cell carcinomas identifies recurrent RAF fusions and frequent inactivation of DNA repair genes. Cancer Discov. 2014; 4:1398-1405. [PubMed: 25266736]

22. Ross JS, Wang K, Chmielecki J, et al. The distribution of BRAF gene fusions in solid tumors and response to targeted therapy. Int J Cancer. 2016; 138:881-890. [PubMed: 26314551]

23. Subbiah V, Westin SN, Wang K, et al. Targeted therapy by combined inhibition of the RAF and mTOR kinases in malignant spindle cell neoplasm harboring the KIAA1549-BRAF fusion protein. J Hematol Oncol. 2014; 7:8. [PubMed: 24422672]

24. Flucke U, van Noesel MM, Wijnen M, et al. TFG-MET fusion in an infantile spindle cell sarcoma with neural features. Genes Chromosomes Cancer. 2017

25. Agaram NP, Zhang L, Sung YS, et al. Recurrent NTRK1 Gene Fusions Define a Novel Subset of Locally Aggressive Lipofibromatosis-like Neural Tumors. Am J Surg Pathol. 2016

26. Haller F, Knopf J, Ackermann A, et al. Paediatric and adult soft tissue sarcomas with NTRK1 gene fusions: a subset of spindle cell sarcomas unified by a prominent myopericytic/haemangiopericytic pattern. J Pathol. 2016; 238:700-710. [PubMed: 26863915]

27. Neubauer K, Zieger B. The Mammalian Septin Interactome. Front Cell Dev Biol. 2017; 5:3. [PubMed: 28224124]

28. Suzuki R, Matsushita H, Kawai H, et al. Identification of a novel SEPT9-ABL1 fusion gene in a patient with T-cell prolymphocytic leukemia. Leuk Res Rep. 2014; 3:54-57. [PubMed: 25068103]

29. Cerveira N, Bizarro S, Teixeira MR. MLL-SEPTIN gene fusions in hematological malignancies. Biol Chem. 2011; 392:713-724. [PubMed: 21714766] 

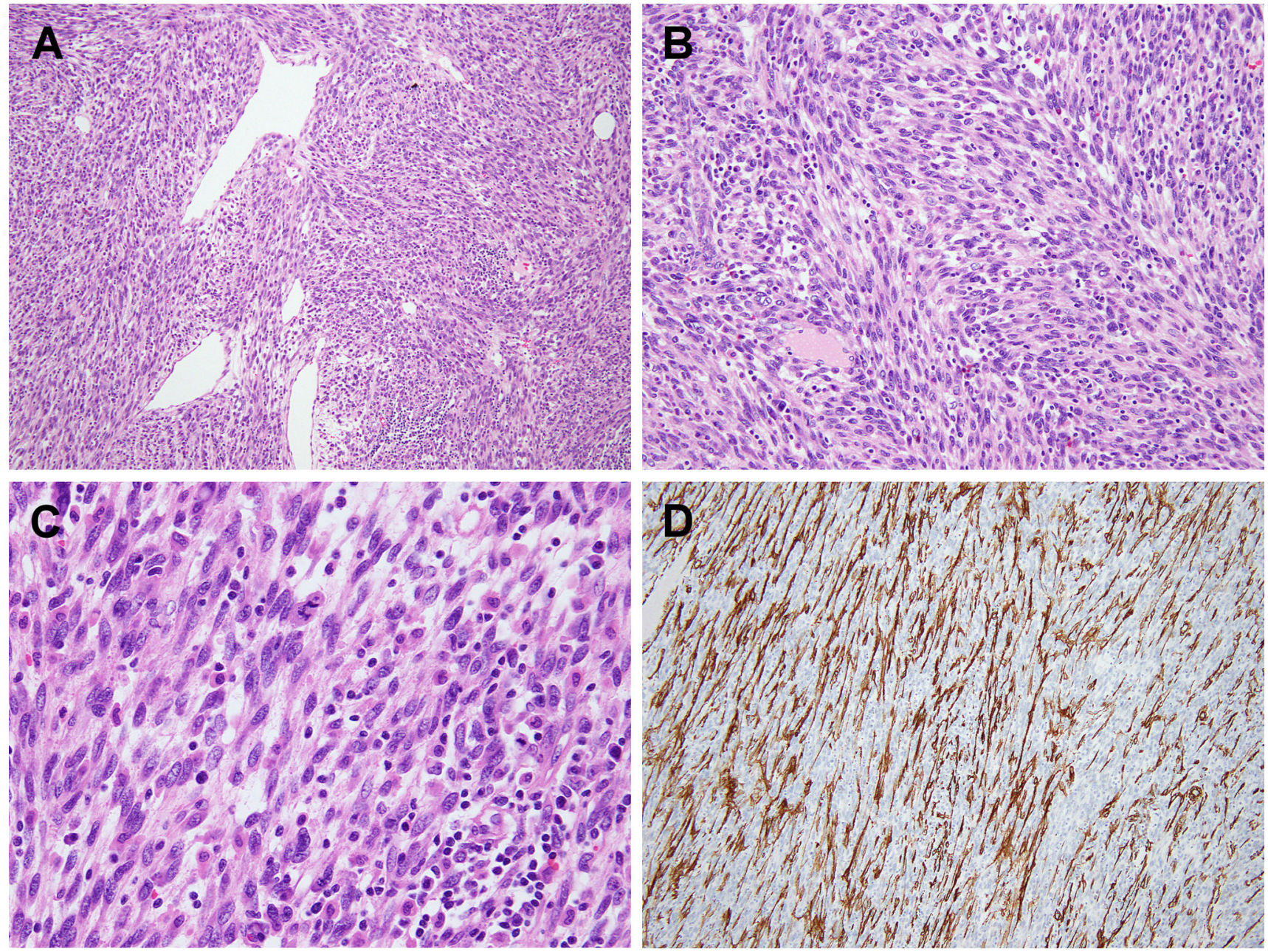

Figure 1.

Histologic features of the index case of a retroperitoneal tumor in a 16 year-old female. The tumor is composed of uniform spindle cells associated with scattered dilated branching vessels (A) and arranged in intersecting fascicles (B). The tumor cells have elongated nuclei, mild atypia, a mitotic count up to 6/10 high power fields, and accompanied by a variable lymphocytic infiltrate (C). Immunohistochemical stains showed focal smooth muscle actin staining (D). 
A.

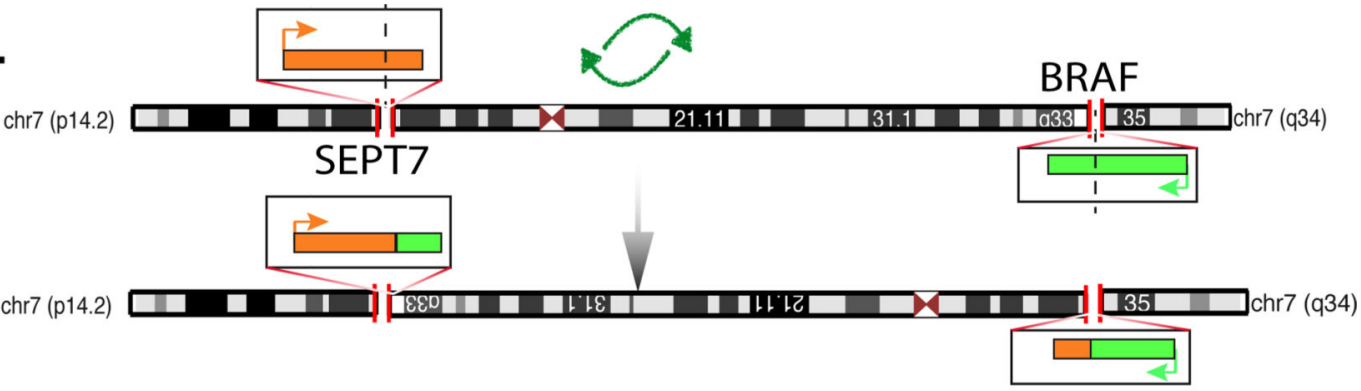

B.

CAAAAACTGAAGGACTCTGAAGCTGAG|AAAACACTTGGTAGACGGGACTCGAGTGATGATTGGGAGATTCCTGAT

TCAAAAACTGAAGGACTCTGAAGCTGAGAAAACACTTGGTAGACGGGACTCGAGTGATGATTGGGAGATTCCTGA AGAAAAAGTTCAAAAACTGAAGGACTCTGAAGCTGAGAAAACACTTGGTAGACGGGACTCGAGTGATGATTGGG AAAGAAAAAGTTAAAAACTGAAGGACTCTGAAGCTGAGAAAACACTTGGTAGACGGGACTCGAGTGATGATCGGG

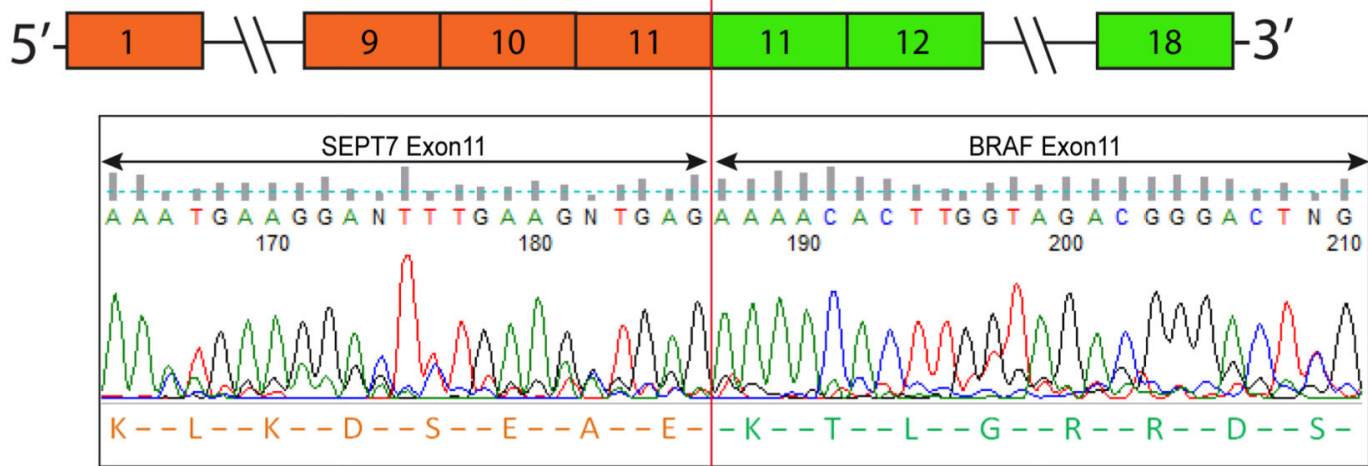

C.
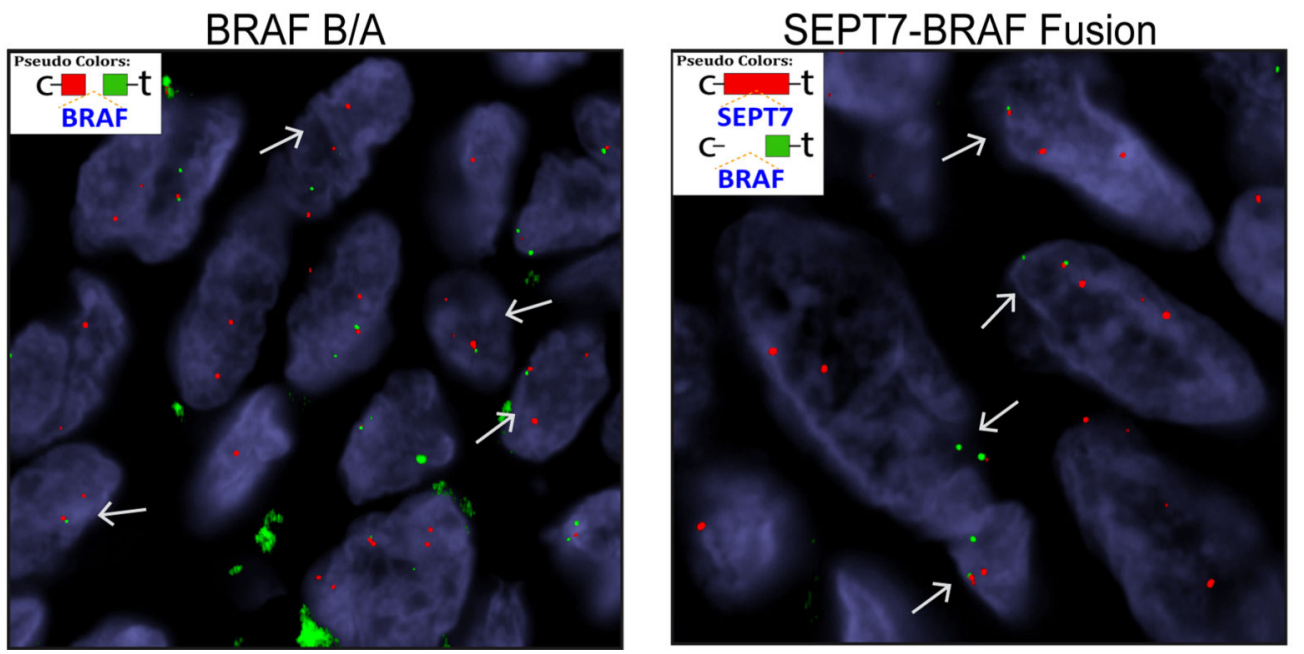

Figure 2.

A $S E P T 7-B R A F$ gene fusion was identified in the index case by targeted RNA sequencing. A pericentric inversion of chromosome 7 resulted in the fusion of $S E P T 7(7 \mathrm{p} 14.2)$ and $B R A F$ (7q34) (A). RNA sequencing fusion junction reads and subsequent confirmatory RTPCR showed $S E P T 7$ exon 11 fused in-frame to $B R A F$ exon 11 (B). FISH further confirmed $B R A F$ gene rearrangement with break-away centromeric (red) and telomeric (green) signals (C, left) as well as $S E P T 7-B R A F$ fusion with come-together $S E P T 7$ (red) and $B R A F$ (green) signals $(\mathrm{C}$, right). 

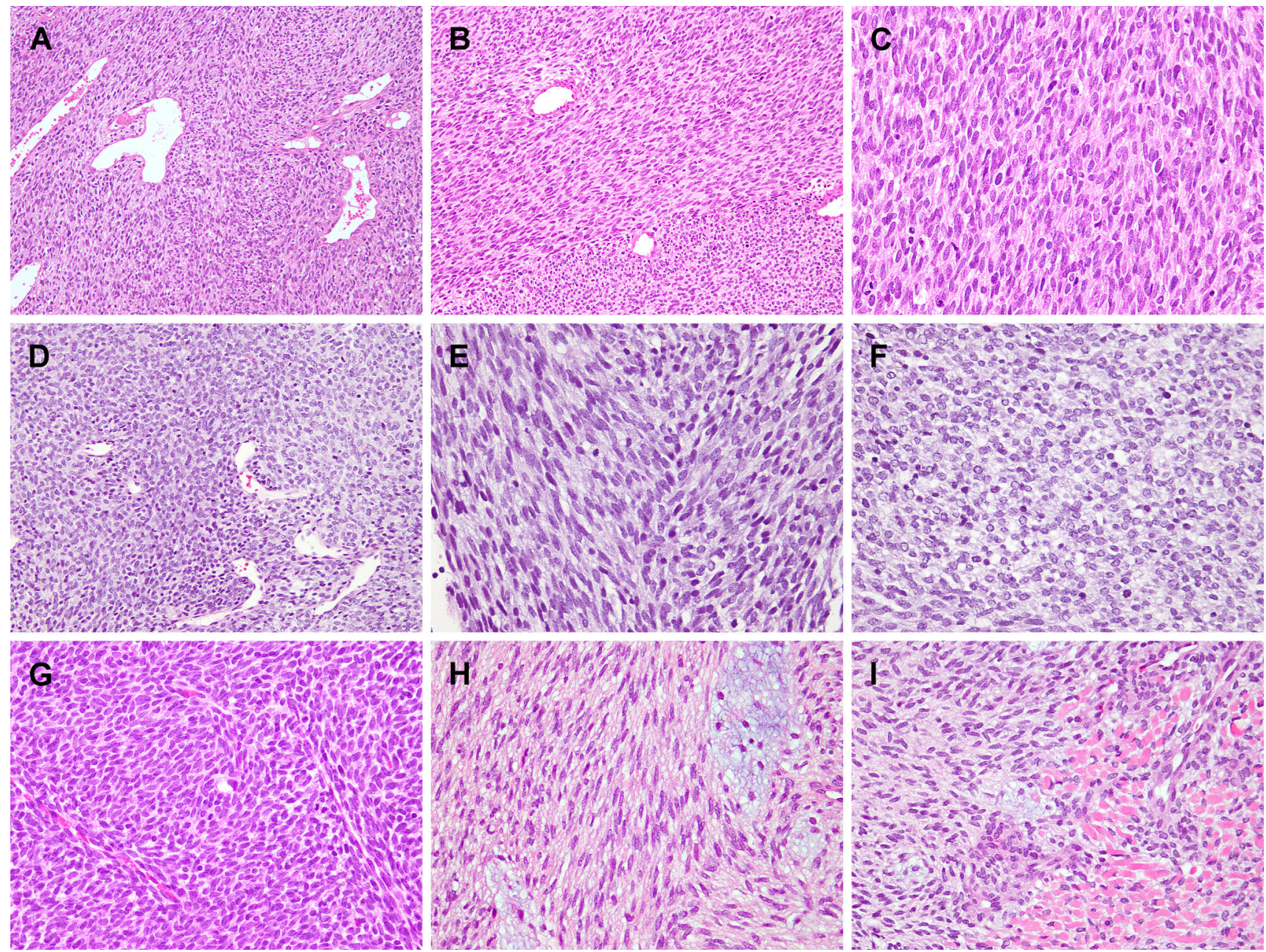

Figure 3.

Histologic features of 4 additional pediatric spindle cell sarcomas resembling IFS with

$B R A F$ rearrangements. Both case 2 (A-C) and case 3 (D-F) occurred in the pelvis, showing a monomorphic spindle cell phenotype arranged in fascicles (B,E) and with scattered dilated vessels (A,D). The tumor cells show fine chromatin pattern (C,E,F). Focal areas of round cell component were also seen in case $3(\mathrm{~F})$. Case $4(\mathrm{G})$, a paraspinal mass, was composed of short spindle to ovoid cells, arranged in dense sheets and focal fascicular growth. Case 5, a congenital thigh mass, showed long spindle cell fascicles with focal myxoid stroma $(\mathrm{H})$ and skeletal muscle infiltration (I). 

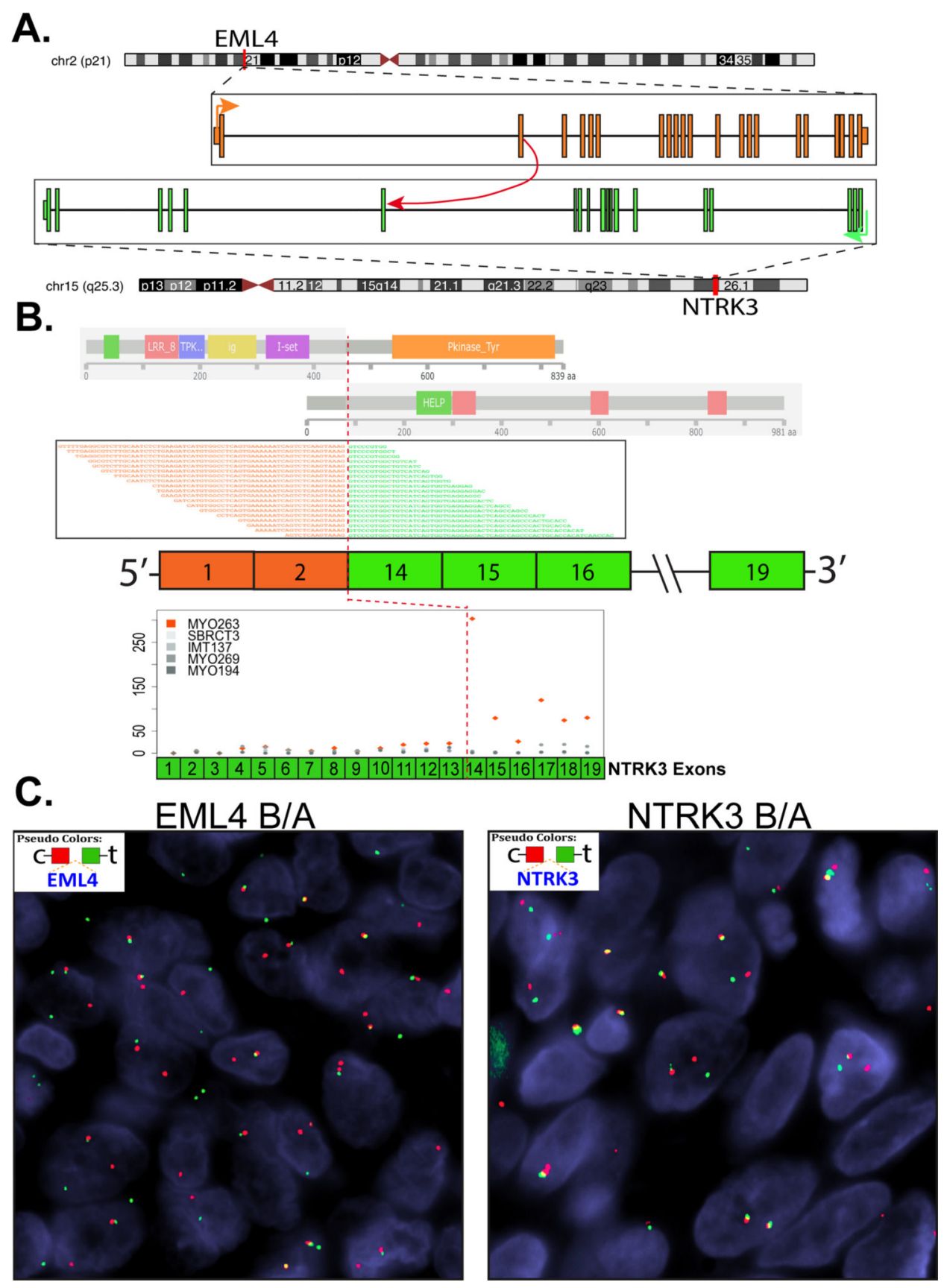

Figure 4.

EML4-NTRK3 fusion in an IFS involving the foot of a 21 month-old boy (case \#6).

Targeted RNA sequencing showed fusion of EML4 exon 2 to NTRK3 exon 14, resulting from a $\mathrm{t}(2 ; 15)$ translocation $(\mathrm{A}, \mathrm{B})$. The chimeric protein contains tyrosine kinase domain from NTRK3 but no known functional domain from EML4 (B). The downstream exons after the breakpoint of $N T R K 3$ (exon 14) showed up-regulated mRNA level in contrast to the 5' $N T R K 3$ exons and other samples in the same dataset (B, orange dots). FISH confirmed rearrangement of both EML4 and NTRK3 genes, with break-apart of centromeric (red) and telomeric (green) signals (C). 
A.

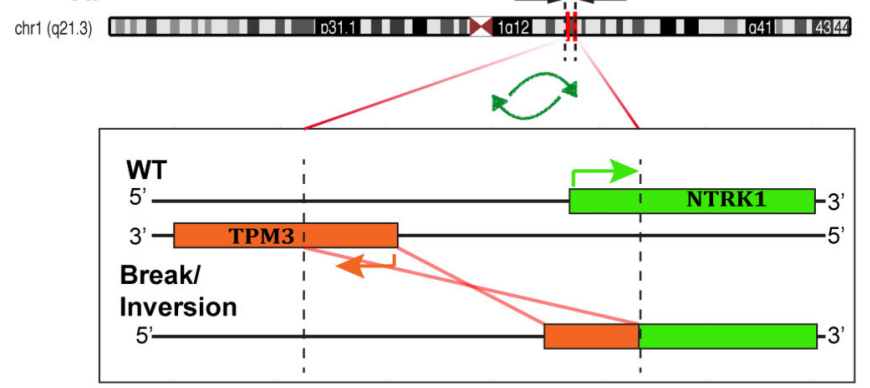

c.

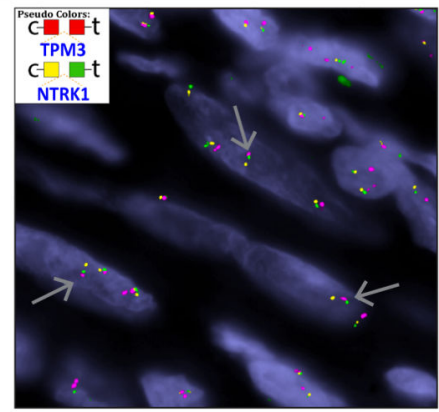

D.
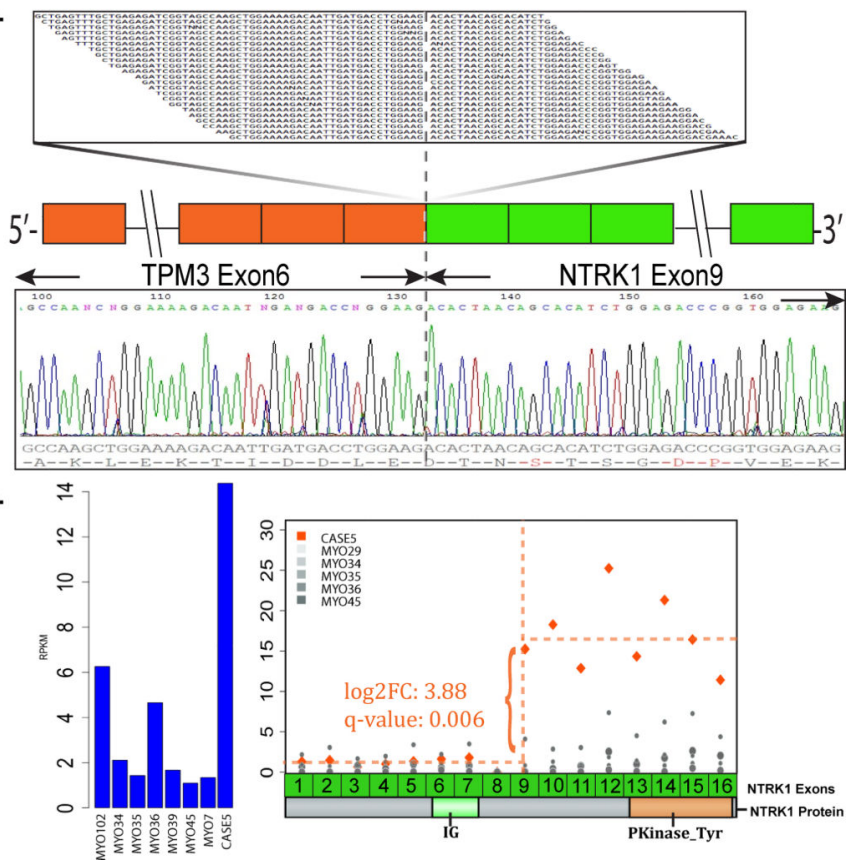

Figure 5.

TPM3-NTRK1 fusion in a 7 week-old infant retroperitoneal IFS (case \#7). Whole transcriptome RNA sequencing identified a $2.7 \mathrm{Mb}$ inversion-fusion at 1q21.3 locus, leading to a TPM3-NTRK1 candidate gene fusion (A). The RT-PCR confirmed an in-frame fusion of TPM3 exon 6 to NTRK1 exon 9 (B). FISH also confirmed NTRK1 rearrangement (split of green and yellow signals) and fusion of TPM3 (red) with NTRK1 (green, telomeric/3' of NTRK1) (C). NTRK1 mRNA expression was up-regulated compared to other samples in the same platform (D, left). Exonic expression levels showed high expression of NTRK1 exons downstream to the break in exon 9; the fusion retaining the entire tyrosine kinase domain in the chimeric protein (D, right). 

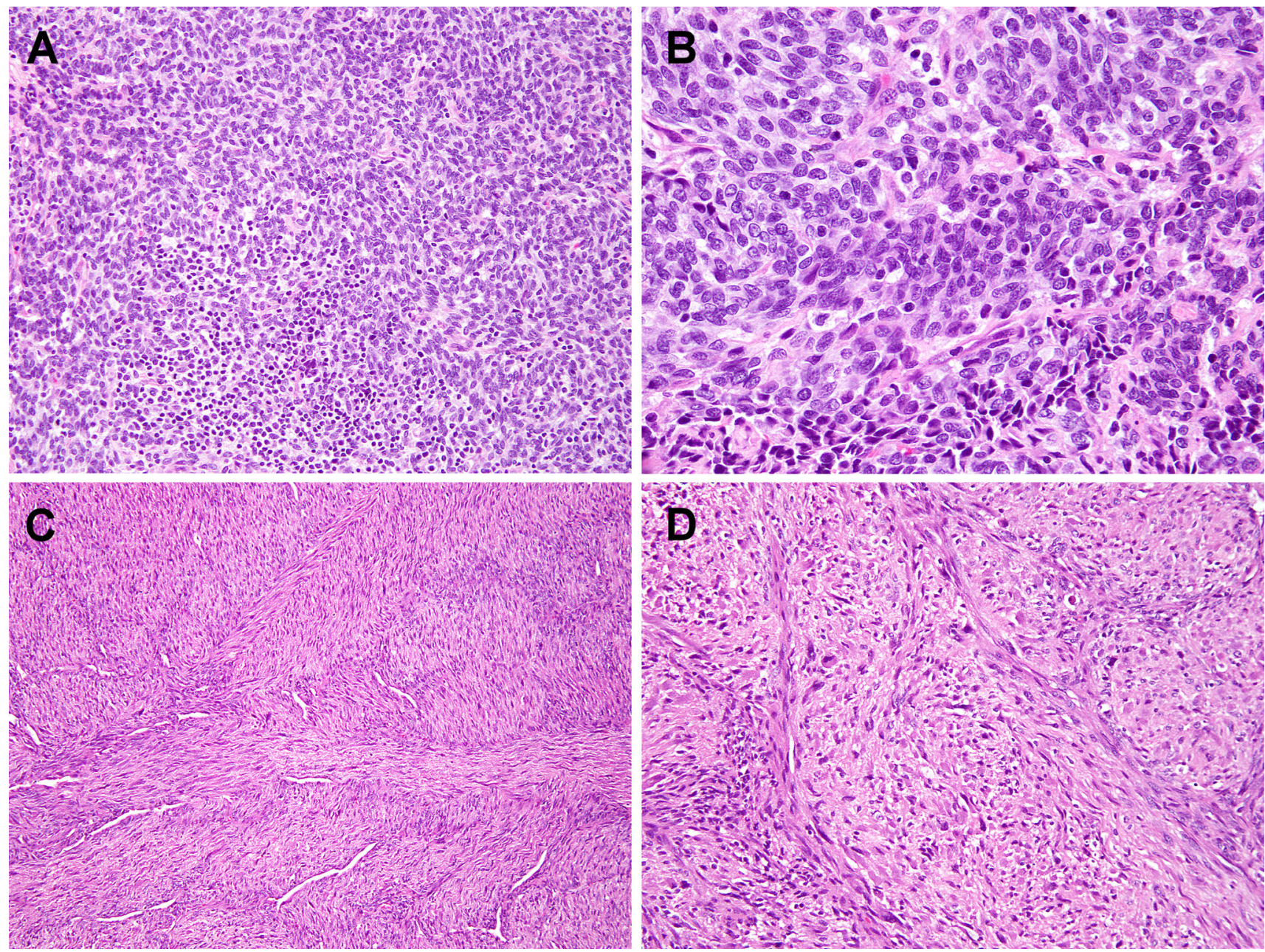

Figure 6.

Histologic features of IFS with EML4-NTRK3 (case \#6; A,B) and TPM3-NTRK1 fusions (case \#7; C,D). Case \#6 showed primitive round, ovoid to short spindle cells with scattered lymphocytic infiltration (A). The tumor cells had monomorphic nuclei, fine chromatin, and scant cytoplasm (B). Case \#7 (primary resection) showed long intersecting fascicles accompanied by cleft-like or compressed vasculature (C, D). The tumor cells had eosinophilic cytoplasm and generally fine chromatin, with scattered atypical hyperchromatic cells (D). 


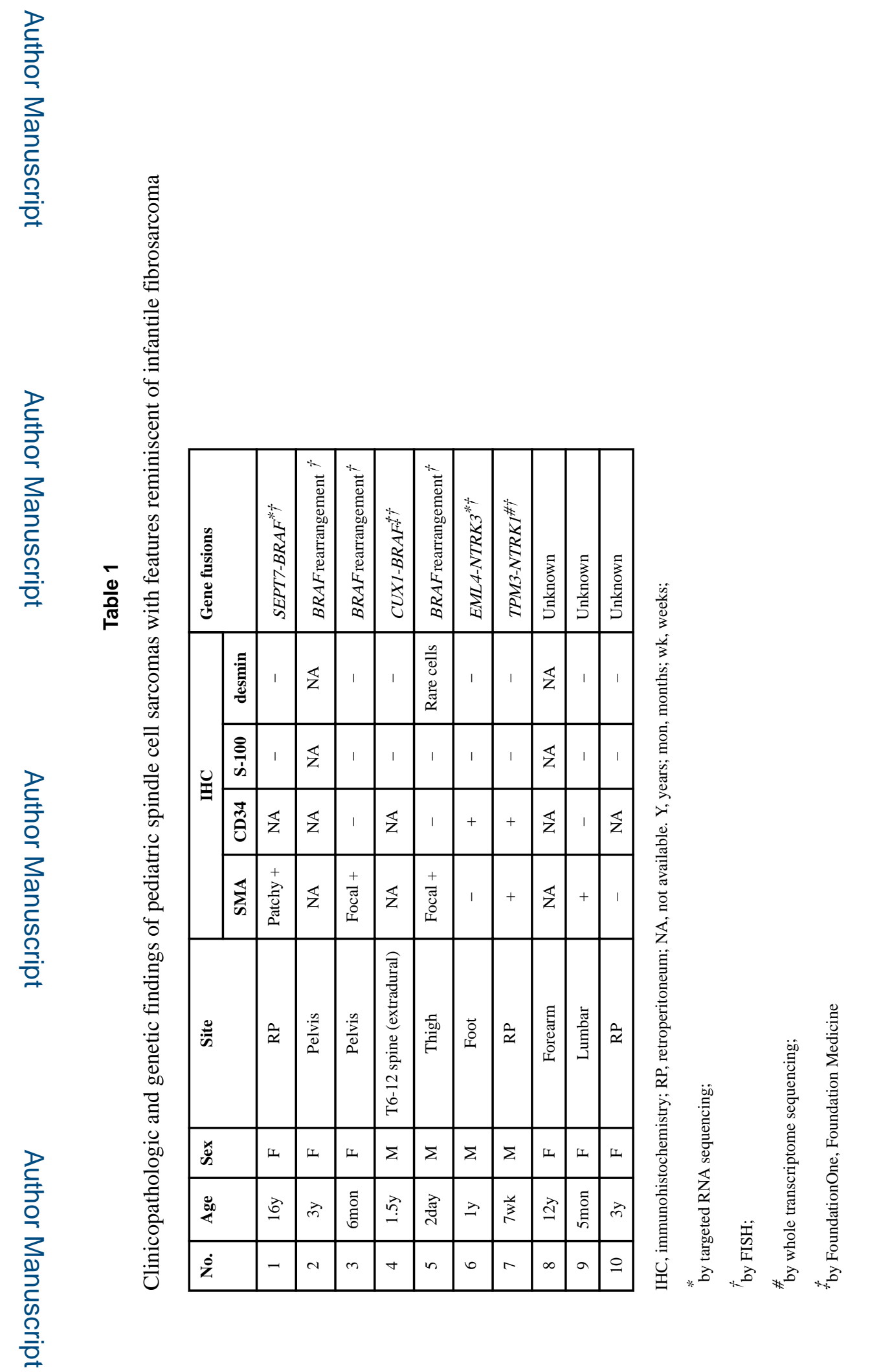

Am J Surg Pathol. Author manuscript; available in PMC 2019 January 01. 